\title{
ChemComm
}



Cite this: Chem. Commun., 2016, 52,11819

Received 30th July 2016, Accepted 2nd September 2016

DOI: $10.1039 / c 6 c c 06313 f$

www.rsc.org/chemcomm

\section{Structure-controlled polymers prepared by pseudo-living addition-condensation polymerization and their application to light harvesting $\dagger$}

\author{
Hao-xuan Guo, Keisuke Yoshida and Hiroyuki Aota*
}

\begin{abstract}
An A,B-block amphiphilic polymer and a branched polymer were prepared by using a new type of pseudo-living addition-condensation polymerization. The first polymer showed an interphase photoinduced energy transfer in a micellar system, while the second type showed efficient light-harvesting ability.
\end{abstract}

Addition-condensation polymerization is generally used in the industrial manufacture of phenol-formaldehyde resins, urea-formaldehyde resins, etc. Poly(pyrrole methine) and its derivatives, which are prepared by the addition-condensation polymerization of pyrrole and different aldehydes, have been studied as narrow-band-gap polymers. ${ }^{1-3}$ In our recent study, we showed the synthesis of water-soluble, narrow-band-gap polymers with band-gap values below $0.19 \mathrm{eV}^{2}$ In addition to being small, the band-gap values of these polymers were continuously controlled from 0.3 to $1.1 \mathrm{eV}$ in aqueous solutions. ${ }^{3}$ The additioncondensation of pyrrole and various aldehydes is also frequently used in the field of porphyrin synthesis. ${ }^{4,5}$ Lindsey et al. have significantly contributed to porphyrin synthesis. In one of their studies, they showed that the $\alpha$-position of dipyrromethane was much more reactive than that of pyrrole. ${ }^{5}$ This observation is very important and interesting for addition-condensation polymerization because it indicates that the reactivities of the dimer, oligomer, and polymer are higher than that of the monomer. The difference in the reactivity of the monomer and other mers may lead to precision polymerization. In general condensationpolymerization reactions, e.g., the synthesis of polyesters and polyamides, the reactivities of the dimer, oligomer, and polymer are almost thermodynamically equal to that of the monomer. Therefore, condensation-polymerization is a form of stepgrowth polymerization, and control of the molecular weight and polymer structure is difficult because of the equal reactivity.

Department of Chemistry and Materials Engineering, Organization for Research and Development of Innovative Science and Technology (ORDIST), Kansai University, Suita, Osaka 564-8680, Japan. E-mail: aota@kansai-u.ac.jp

$\dagger$ Electronic supplementary information (ESI) available. See DOI: 10.1039/ c6cc06313f
In contrast, precision polymerizations have been studied in living anionic, ${ }^{6}$ living cationic, ${ }^{7}$ living free-radical, ${ }^{7 c, 8}$ and living ring-opening metathesis polymerizations. ${ }^{9}$ These polymerizations are a form of chain-growth polymerization. Herein, living chaingrowth polycondensation was studied as an example of special precision polymerization. ${ }^{10}$ In this condensation-polymerization, the reactivity between the polymer end group and the monomer is higher than that between a monomer and monomer. Therefore, the monomers preferentially react with the polymer end groups. In other words, the difference in the reactivity of the monomer and the other mers in the addition-condensation polymerization of pyrrole and aldehyde leads to precision polymerization, which allows for the control of the main structural properties of the polymer as well as the introduction of functional groups (e.g., energy donors and acceptors) by the addition of different aldehydes.

Photoinduced energy transfer, especially light harvesting, has been studied in both natural and artificial photosynthetic systems. For example, light harvesting occurs in the photosynthetic reaction center ${ }^{11}$ and has been studied in porphyrins,${ }^{12}$ polymers, ${ }^{13}$ and dendrimers ${ }^{14}$ in order to construct an artificial photosynthetic system.

In this paper, we show the pseudo-living addition-condensation polymerization of 1-methylpyrrole (MePyr) and an aldehyde. We used MePyr instead of pyrrole because the resulting polymer prepared from MePyr is stable in air while that of pyrrole is not. Two types of structure-controlled polymers were prepared. The first is an A,B-block amphiphilic polymer with energy donors and acceptors, each in a different block. This type of polymer showed an interphase photoinduced energy transfer in a micellar system. The second polymer is a branched polymer with high and low amounts of energy donors and acceptors, respectively. This type of polymer showed an efficient light-harvesting ability.

The rate-determining step in the addition-condensation of MePyr and aldehyde in the presence of acid is the addition of aldehyde to the $\alpha$-position of MePyr, which is shown in step- 1 in Fig. 1b and Fig. S1a (ESI†). The reactivity of the subsequent condensation, step-2 in Fig. 1b, is higher than that of step-1. 


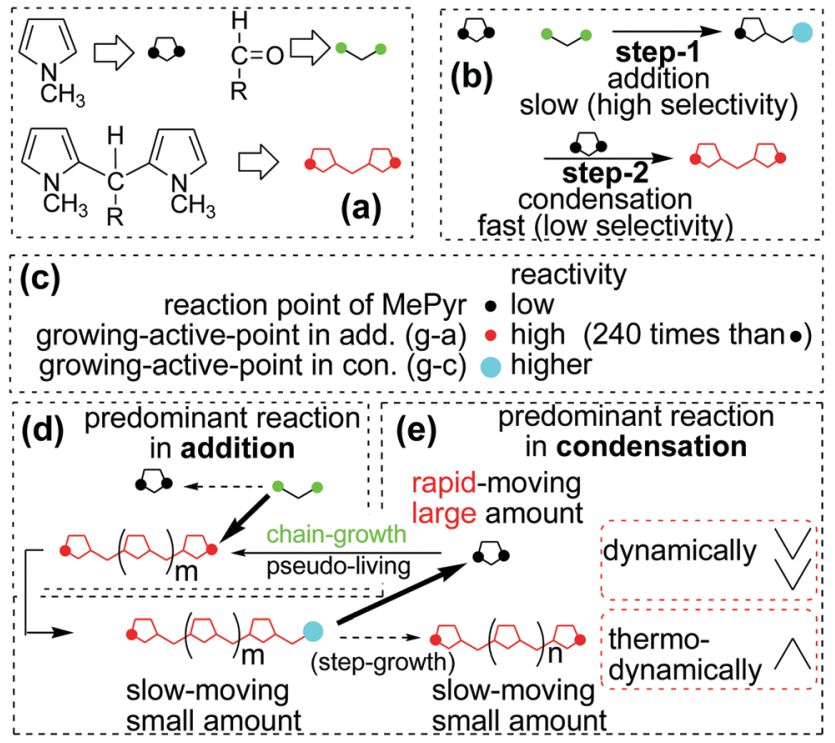

Fig. 1 Reaction mechanism of the pseudo-living addition-condensation polymerization of 1-methylpyrrole and aldehyde.

Therefore, the addition occurs with high selectivity. Moreover, the electron density at the $\alpha$-position of the generated dimer, dipyrromethane, is higher than that of the monomer, MePyr. As a result, the reactivity at the $\alpha$-position of the generated dipyrromethane is 240 times higher than that of MePyr at $10{ }^{\circ} \mathrm{C}$ (shown in Fig. S2, ESI $\dagger$ ). When $1 \%$ of dipyrromethane is generated in the initial stage of the polymerization, the reactivity ratio of dipyrromethane to MePyr becomes about $2.4: 1$. In other words, the growing-active-point in addition ( $\mathrm{g}-\mathrm{a}$ ) increases during the initial stage of polymerization ( $\mathrm{g}$-a is defined as the $\alpha$-position of the dimer (dipyrromethane), the oligomer, or the polymer (shown as a red-dot in Fig. 1)). However, the g-a immediately becomes almost constant at low concentrations because the propagation reaction of the $\mathrm{g}$-a with aldehyde occurs much faster than the reaction of MePyr with aldehyde (shown in Fig. 1d); thus, the generation of new g-a is limited because the reaction of MePyr with aldehyde is so slow.

In the condensation, the growing-active-point in condensation (g-c, shown as a blue-dot in Fig. 1) is defined as the carbon with the attached hydroxyl group, which is generated by the addition of aldehyde. The reaction of the g-c with the g-a is thermodynamically preferred. However, this reaction is hindered by the following means: the higher reactivity of the g-c lowers the selectivity. Therefore, the collision of the g-c with MePyr becomes more significant. In the initial and middle stages of the polymerization, the $\mathrm{g}$-c is at lower concentrations like the $\mathrm{g}-\mathrm{a}$, which was mentioned above. In contrast, a high concentration of MePyr is present in these stages. Moreover, the polymers move slowly because of their high molecular weight (MW); therefore, collisions between the polymers are very rare while collisions between the polymer and MePyr occur frequently due to the high concentration and relatively rapid movement of MePyr. Ultimately, the propagation reaction occurs dynamically and more frequently than the polymer-polymer reaction (shown in Fig. 1e). Therefore, the addition-condensation of MePyr with aldehyde progresses like a chain-growth polymerization in the initial and middle stages. Finally, the polymerpolymer reaction occurs in the final stage of polymerization (shown in Fig. S3a(iii), ESI $\dagger$ ) because of the low concentration of MePyr. These concepts agree with the experimental results obtained for the polymerization of MePyr and the equimolar benzaldehyde-2-sulfonic acid sodium salt (BS) (mentioned in the ESI $\uparrow$ (Fig. S4)).

The control of the amount of MePyr is important in this polymerization. We controlled the MePyr amount as follows:

Initial stage: aldehyde was slowly added to an excess of MePyr. In this stage, the dimer (dipyrromethane) and the oligomer were generated (shown in Fig. S3b(i), ESI $\dagger$ ).

Progress stage 1: the aldehyde was continuously added until the added amount was half that of MePyr (shown in Fig. S3b(ii), ESI $\dagger$ ). The added aldehyde, which had rapid movement due to its low MW, reacted at the g-a predominantly, then the generated g-c was immediately attacked by the large amount of MePyr and became the larger g-a (illustrated in Fig. 1d and e). Moreover, this immediate reaction of the g-c with MePyr is important as it prevents the termination, which occurs by the side reaction shown in Fig. S1d (ESI $\dagger$ ).

Progress stage 2: after the addition of a half equivalent of aldehyde, a 1:1 mixture of both aldehyde and MePyr was continuously added (shown in Fig. S3b(iii), ESI $\dagger$ ). The ratio of $\mathrm{g}$-a to MePyr was kept nearly constant during the polymerization. The polymer-polymer reaction was prevented by both the sluggish mobility of the high MW polymer and the high concentration of MePyr, which had relatively free movement due to its low MW. Thus, the propagation reaction in this stage proceeds like a livingpolymerization with the continuous addition of both monomers.

Fig. S5 (ESI $\dagger$ ) shows the MW-time curve of the polymerization of MePyr and BS with the continuous addition of both monomers. Here, progress stage 2 was carried out after 1 day of initiation. The linear relationship between the MW and the added amount of monomers means that this polymerization progresses like a living-polymerization. ${ }^{6-10}$ A rough estimate of the molecular weight distribution of the polymer obtained at 8 days was 1.5. This higher value means that the polymerization is a pseudo-living rather than an intrinsic living-polymerization (the value of the latter should be nearly 1.0). The details of the polymerization are provided in the ESI. $\dagger$

Many types of aldehydes can be used in this polymerization. The controlled addition of certain aldehydes leads to structure-controlled polymers. There are four different concepts for the structure control. (1) The molecular weight is controlled by changing the continuous addition time (shown in Fig. S5, ESI†). (2) The polymer's polarity is controlled by using hydrophobic and hydrophilic aldehydes; e.g., a water-soluble polymer is easily prepared by using anionic aldehyde, and an A,B-block amphiphilic polymer is prepared by the sequential addition of hydrophobic and hydrophilic aldehydes. (3) The branched and cross-linked polymers are prepared using 1-methyl2-pyrrolecarboxaldehyde and terephthalaldehyde. (4) Functional groups, e.g., the energy donors and acceptors, are introduced into the polymer chain using the functional aldehydes. 
Following the previous guidelines, we prepared two types of polymers to study the photoinduced energy transfer and their ability to behave as light-harvesting materials in order to construct an artificial photosynthetic system.

The structures of the aldehydes and 2-ethylpyrrole (2-EtPyr), which was used as the terminal unit, are shown in Fig. 2a. The reactivity ratio of 2-EtPyr to MePyr is 64:1 and is discussed in detail in the ESI $\dagger$ (Fig. S2). Therefore, 2-EtPyr reacted in the initiation stage, and the polymer grew linearly in one direction. The image of preparation and the schedule of addition are shown in Fig. 2b and Fig. S6 (ESI†), respectively. The sequential addition of monomers to 2-EtPyr and an excess amount of MePyr was conducted in the following order: step (1), [4-isopropylbenzaldehyde (iPB) and 1-pyrenecarboxaldehyde (PyA)]; step (2), [iPB]; step (3), [BS and MePyr]; and step (4), [BS, MePyr, and 3-perylenecarboxaldehyde (PeA)]. The details are provided further in the ESI. $\dagger$

The ability for a reaction to occur at the interphase is an important component in the construction of an artificial photosynthetic system because it allows for efficient separation of high energy substances generated by photochemical reactions. ${ }^{15}$ A fluorescence quenching method was used to provide evidence

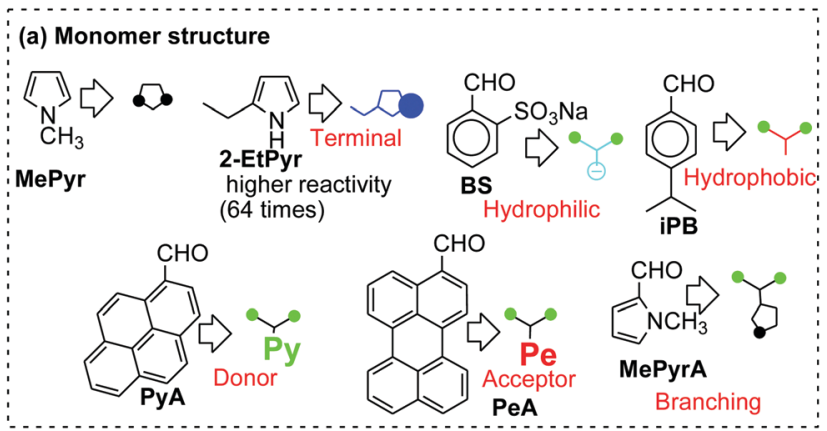

(b) A, B-block amphiphilic polymer for interphase photoinduced
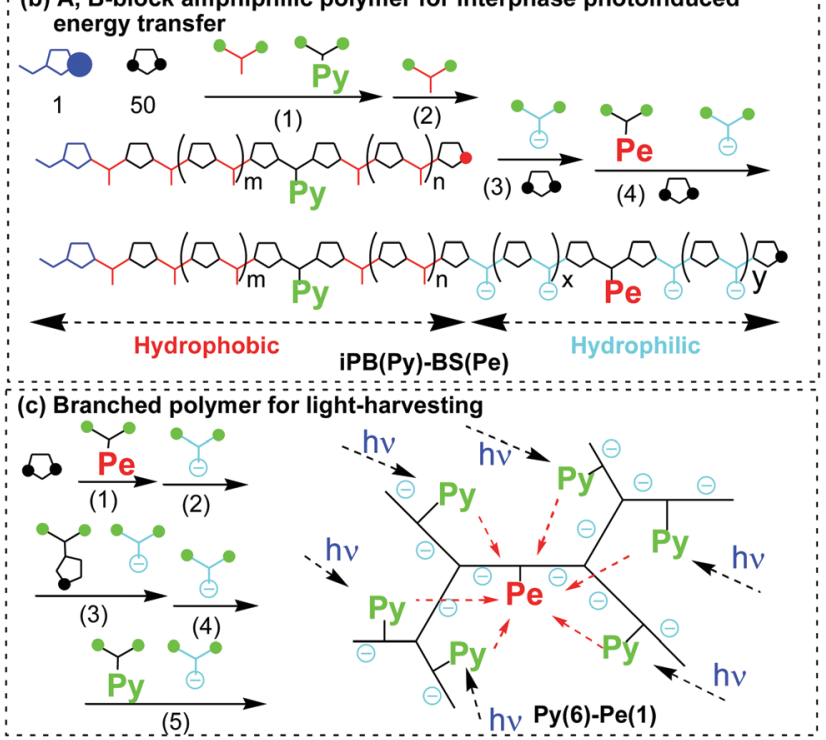

Fig. 2 Preparation of structure-controlled polymers by pseudo-living addition-condensation polymerization by the continuous addition of monomers.
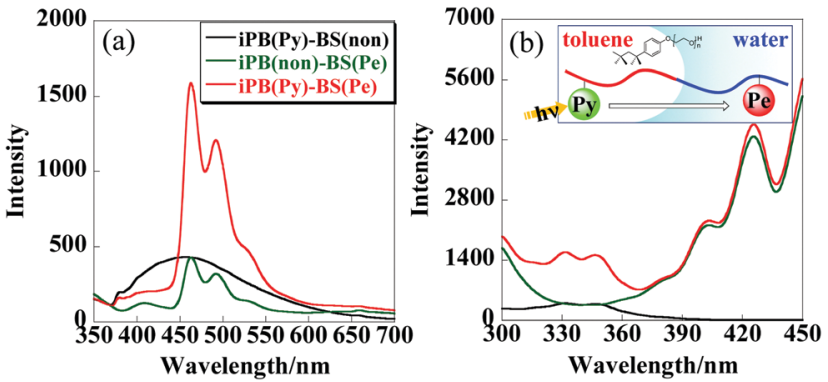

Fig. 3 (a) Fluorescence and (b) excitation spectra of the A,B-block amphiphilic polymers containing $\mathrm{Py}$ and $\mathrm{Pe}$ units dissolved in a water/ toluene emulsion (118/1) including $1.8 \%$ Triton $\mathrm{X}$ at $25^{\circ} \mathrm{C}$, [polymer] $=$ $0.083 \mathrm{~g} \mathrm{~L}^{-1}$, (a) excited at $330 \mathrm{~nm}$, (b) monitored at $460 \mathrm{~nm}$, cell length $=1 \mathrm{~cm}$.

that the prepared polymer exists at the heterophase boundary with the donor and acceptor groups positioned in the two different phases (shown in the inset in Fig. 3b). The details are provided in the ESI $\dagger$ (Fig. S8 and S9, ESI $\dagger$ ). Three reference polymers were made for this purpose: $\mathrm{iPB}(\mathrm{Py})-\mathrm{BS}(\mathrm{Pe})$, iPB(Py)$\mathrm{BS}($ none), and $\mathrm{iPB}($ none)-BS(Pe). The abbreviation iPB(Py)$\mathrm{BS}(\mathrm{Pe})$ means that the Py unit is in the hydrophobic phase and the Pe unit is in the hydrophilic phase. Fig. 3a and b and Fig. S10 (ESI $\dagger$ ) show the fluorescence, excitation, and absorption spectra of the polymers, respectively. In the fluorescence spectra $\left(\lambda_{\text {exc }}=330 \mathrm{~nm}\right)$ in which the Py unit dominates, the fluorescence intensity of $\mathrm{iPB}(\mathrm{Py})-\mathrm{BS}(\mathrm{Pe})$, which emits around $460 \mathrm{~nm}$, is higher than that of iPB(none)-BS(Pe). Conversely, the intensity of $\mathrm{PB}(\mathrm{Py})-\mathrm{BS}(\mathrm{Pe})$, which emits around $400 \mathrm{~nm}$, is lower than that of $\mathrm{iPB}(\mathrm{Py})-\mathrm{BS}($ none). This strongly suggests that the interphase photoinduced energy transfer from the excited Py unit to the Pe unit occurs in the micellar system. In the excitation spectra monitored at $460 \mathrm{~nm}$ in which the Pe unit dominates, the intensity at around $330 \mathrm{~nm}$ of $\mathrm{PBB}(\mathrm{Py})-\mathrm{BS}(\mathrm{Pe})$ is higher than that of the other polymers. This result confirms the occurrence of the interphase photoinduced energy transfer.

The structures of the aldehydes and 1-methyl-2-pyrrolecarboxaldehyde (MePyrA), which is used as a branching unit, are shown in Fig. 2a. The image of preparation and the schedule of addition are shown in Fig. 2c and Fig. S7 (ESI $\dagger$ ), respectively. The sequential addition to an excess of MePyr was conducted in the following order: step (1), [PeA (at one time addition)]; step (2), [BS]; step (3), [BS and MePyrA]; step (4), [BS]; and step (5), [BS and PyA]. The details are provided in the ESI. $\dagger$

Light harvesting is the initial step of natural photosynthesis. ${ }^{11}$ Fig. $4 \mathrm{a}$ and $\mathrm{b}$ and Fig. S11 (ESI $\dagger$ ) show the fluorescence, excitation, and absorption spectra of the prepared polymers, respectively. The abbreviation Py(6)-Pe(1) indicates that the molar ratio of the Py unit to the Pe unit is $6: 1$. The structural image of $\mathrm{Py}(6)-\mathrm{Pe}(1)$ is shown in Fig. 2c. The absorbance around $330 \mathrm{~nm}$ increased with respect to the relative amount of the Py unit (shown in Fig. S11, ESI $\dagger$ ). The fluorescence intensity around $460 \mathrm{~nm}$ (the emission of the Pe unit) increased almost linearly with respect to the relative amount of the Py unit (shown in Fig. 4a). This indicates that the photoinduced energy transfer occurs from the multi donors to one acceptor. In the excitation spectra monitored at 

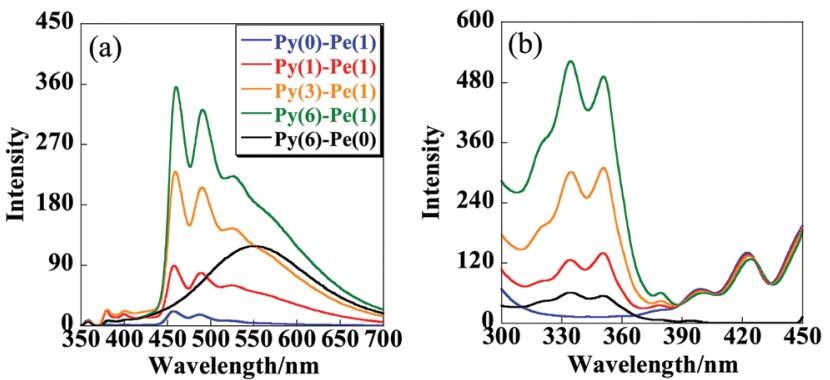

Fig. 4 (a) Fluorescence and (b) excitation spectra of branched polymers containing high and low amounts of Py and Pe units dissolved in water at $25{ }^{\circ} \mathrm{C}$, abs $(420 \mathrm{~nm})=0.01$, except $\mathrm{Py}(6)-\mathrm{Pe}(0)$, (a) excited at $320 \mathrm{~nm}$, (b) monitored at $455 \mathrm{~nm}$, cell length $=1 \mathrm{~cm}$.

$455 \mathrm{~nm}$, the intensity around $330 \mathrm{~nm}$ increases with respect to the relative amount of the Py unit. This indicates an efficient light-harvesting mechanism.

In summary, we have shown a new type of pseudo-living polymerization by continuous addition of monomers to the addition-condensation of 1-methylpyrrole and aldehydes. Typically, precision polymerizations, which allow for structure-controlled polymers, are very tedious because they require severe experimental conditions. However, our new synthesis method does not require any special precautions. Additionally, this pseudoliving addition-condensation polymerization is able to both control the polymer main structures and introduce functional groups by the sequential addition of different aldehydes. Two types of polymers were prepared to construct an artificial photosynthetic system using this polymerization. The A,B-block amphiphilic polymer showed an interphase photoinduced energy transfer in a micellar system, and the branched polymer indicated an efficient light-harvesting ability. We conclude that this new pseudo-living addition-condensation polymerization will yield high-performance polymers that may be used in many scientific fields.

The early part of this study was contributed by K. Watanabe, A. Murata, E. Yokoi, E. Ichihashi, Y. Uchida, S. Nishimura, Y. Kondo, Y. Hamamura, S. Suzuki, Y. Akiyama, and Y. Nakamura. The authors thank Emeritus Prof. A. Matsumoto for his shrewd advice. H. A. would like to specially thank D. Gust at Arizona State University for providing us with an opportunity to study artificial photosynthesis and photoenergy conversion as a Kansai University overseas research fellow. This work was supported by a Grant-in-Aid for Exploratory Research (No. 15656244, 23656608), HAITEKU, and the Strategic Research Foundation at Private Universities from the Ministry of Education, Culture, Sports, Science and Technology
(MEXT). This research was also financially supported by the Kansai University Grant-in-Aid for progress of research in graduate course 2014, 2015, and 2016.

\section{References}

1 (a) R. Becker, G. Blöchl and H. Bräunling, NATO ASI Ser., Ser. E, 1990, 182, 133; (b) H. Bräunling, R. Becker and G. Blöchl, Synth. Met., 1991, 42, 1539; (c) H. Goto and K. Akagi, J. Polym. Sci., Part A: Polym. Chem., 2005, 43, 616; (d) H. Aota, Y. Itai, A. Matsumoto and M. Kamachi, Chem. Lett., 1994, 2043; (e) H. Aota, T. Reikan, A. Matsumoto and M. Kamachi, Chem. Lett., 1997, 527; $(f)$ J. M. Toussaint, B. Thémans, J. M. André and J. L. Brédas, Synth. Met., 1989, 28, 205; $(g)$ F. Meyers, C. Adant, J. M. Toussaint and J. L. Brédas, Synth. Met., 1991, 43, 3559.

2 H. Aota, T. Ishikawa, Y. Amiuchi, H. Yano, T. Kunimoto and A. Matsumoto, Chem. Lett., 2010, 39(12), 1288.

3 H. Aota, T. Ishikawa, Y. Maki, D. Takaya, H. Ejiri, Y. Amiuchi, H. Yano, T. Kunimoto and A. Matsumoto, Chem. Lett., 2011, 40, 724.

4 (a) P. Rothemund, J. Am. Chem. Soc., 1936, 58, 625; (b) A. D. Adler, F. R. Longo, J. D. Finarelli, J. Goldmacher, J. Assour and L. Korsakoff, J. Org. Chem., 1967, 32(2), 476; (c) J. S. Lindsey, I. C. Schreiman, H. C. Hsu, P. C. Kearney and A. M. Marguerettaz, J. Org. Chem., 1987, 52(5), 827.

5 C.-H. Lee and J. S. Lindsey, Tetrahedron, 1994, 50(39), 11427.

6 (a) M. Szwarc, M. Levy and R. Milkovich, J. Am. Chem. Soc., 1956, 78(11), 2656; (b) Y. Kohsaka, T. Kurata, K. Yamamoto, S. Ishihara and T. Kitayama, Polym. Chem., 2015, 6, 1078.

7 (a) M. Sawamoto, J. Fujimoro and T. Higashimura, Macromolecules, 1987, 20, 916; (b) P. De and R. Faust, Macromolecules, 2005, 38, 9897; (c) H. Aoshima, M. Uchiyama, K. Satoh and M. Kamigaito, Angew. Chem., Int. Ed., 2014, 53(41), 10932.

8 (a) J. K. Kochi, J. Am. Chem. Soc., 1956, 78, 4815; (b) J. S. Wang and K. Matyjaszewski, J. Am. Chem. Soc., 1995, 117, 5614; (c) W. Tang and K. Matyjaszewski, Macromolecules, 2007, 40, 1858; (d) W. A. Braunecker and K. Matyjaszewski, Prog. Polym. Sci., 2007, 32, 93.

9 (a) N. J. Calderon, J. Macromol. Sci. Rev. Macromol. Chem., 1972, 7, 105; (b) R. R. Schrock, J. Feldman, L. F. Cannizzo and R. H. Grubbs, Macromolecules, 1987, 20(5), 1169; (c) T. Aida, Prog. Polym. Sci., 1994, 19, 469; (d) C. W. Bielawski and R. H. Grubbs, Prog. Polym. Sci., 2007, 32, 1.

10 (a) D. Robello, A. Ulman and E. Urankar, Macromolecules, 1993, 26, 6718; (b) T. Yokozawa, T. Asai, R. Sugi, S. Ishigooka and S. Hiraoka, J. Am. Chem. Soc., 2000, 122, 8313; (c) T. Yokozawa, D. Muroya, R. Sugi and A. Yokoyama, Macromol. Rapid Commun., 2005, 26, 979; (d) T. Yokozawa and A. Yokoyama, Prog. Polym. Sci., 2007, 32, 147.

11 (a) G. McDermott, S. M. Prince, A. A. Freer, A. M. HawthornthwaiteLawless, M. Z. Papiz, R. J. Cogdell and N. W. Isaacs, Nature, 1995, 374, 517; (b) W. Kühlbrandt, Nature, 1995, 374, 497.

12 (a) J. S. Lindsey, P. A. Brown and D. A. Siesel, Tetrahedron, 1989, 45, 4845-4866; (b) A. Satake and Y. Kobuke, Org. Biomol. Chem., 2007, $5,1679$.

13 S. E. Webber, Chem. Rev., 1990, 90, 1469.

14 (a) D.-L. Jiang and T. Aida, J. Am. Chem. Soc., 1998, 120, 10895; (b) S. Cho, W.-s. Li, M.-C. Yoon, T. K. Ahn, D.-L. Jiang, J. Kim, T. Aida and D. Kim, Chem. - Eur. J., 2006, 12, 7576.

15 (a) G. Steinberg-Yfrach, P. A. Liddell, S.-C. Hung, A. L. Moore, D. Gust and T. A. Moore, Nature, 1997, 385, 239; (b) G. Steinberg-Yfrach, J.-L. Rigaud, E. N. Durantini, A. L. Moore, D. Gust and T. A. Moore, Nature, 1998, 392, 479. 